\title{
Fatores que Influenciam os Parâmetros das Curvas de Lactação em Cabras no Distrito Federal ${ }^{1}$
}

\section{Concepta McManus ${ }^{2}$, Guilherme Soares Filho $^{3}$, Arthur da Silva Mariante ${ }^{4}$, Helder Louvandini ${ }^{2}$}

\begin{abstract}
RESUMO - O objetivo deste trabalho foi avaliar a influência de fatores ambientais sobre a forma de curvas de lactação (Quadrática, Gama, Linear Hiperbólica e Jenkins e Ferrel) de cabras das raças Saanen, Parda Alpina, Toggenburg e seus mestiços. Foram usados registros de 299 lactações de 215 cabras criadas em dois criatórios localizados no Distrito Federal no período de 1995 a 1997. Os parâmetros das curvas de lactação (produção no início da lactação - $a$, taxa média de ascenção - $b$ e taxa média de declínio após o pico - $c$ ) foram estimados pelo programa "Table Curve" da companhia de Jandel Scientific. Estes parâmetros foram analisados usando o programa SAS com os procedimentos Corr e GLM. O modelo estatístico incluiu como fatores fixos o capril, a raça, o mês e o ano de parto, tipo de parto, sexo do cabrito, e o peso de cobertura foi utilizado como covariável. Os parâmetros $a$ e $c$ foram influenciados pela raça, pelo mês do parto e pelo tipo do parto. As curvas Quadrática e Linear Hiperbólica tiveram os maiores $\mathrm{R}^{2}$. As correlações entre os parâmetros das curvas e os índices zootécnicos, no geral, foram altas e positivas. Este estudo mostra que qualquer uma das funções estudadas poderia representar a curva de lactação individual do rebanho, no entanto, a que melhor expressou os parâmetros na formação da curva foi a função Quadrática, enquanto aquelas que melhor descreveram a produtividade foram o Linear Hiperbólica e Jenkins e Ferrel.
\end{abstract}

Palavras-chave: fatores ambientes, "goodness of fit", parâmetros

\section{Factors which Influence Parameters of Goat Lactation Curves in the Federal District}

ABSTRACT - The objetive of this work was to evaluate the effect of environmental factors on the shape of lactation curves (Quadratic, Gama, Linear Hiperbolic and Jenkins \& Ferrel) of Saanen, Alpine and Toggenburg as well as mixed breed goats. Data from 299 lactations of 215 goats raised on two farms in the Federal District from 1995 to 1997 was used. The parameters of the lactation curves (yield at start of lactation- $a$, rate of increase of milk production - $b$ e rate of decline of milk production after peak rate - $c$ ) were estimated using the Table Curve program from Jandel Scientific. The parameters were then analysed using SAS (Statistical Analysis System) (CORR and GLM procedures). The statistical model included fixed effects (farm, breed, month and year of kidding, type of kidding, kid sex) and covariates (weight at insemination). Parameters $a$ and $c$ were influenced by breed, month of kidding as well as type of kidding. The Quadratic and Linear Hyperbolic curves had the highest $\mathrm{R}^{2}$. The correlations between curve parameters and production indices were, in general, high and positive. This study shows that any in the functions studied may be used to represent the individual lactation curve of herd, however, the curve that best expressed the shape of the lactation curve for goats was the Quadratic function while the Linear Hyperbolic and Jenkins and Ferrel had higher correlation between total production and curve parameters.

Key Words: enviromental factors, goodness of fit, parameters

\section{Introdução}

A curva de lactação é um importante estudo que possibilita a identificação de possíveis falhas de manejo de um determinado rebanho, como alimentação deficiente, instalações inadequadas, patologias não aparentes, entre outras (Graminha et al., 1996). Permite também que seja acompanhada a evolução da produção leiteira dos animais, com o conhecimento de suas variações ao longo de uma lactação, avaliando um animal ou um grupo deles, estimando sua produção de leite parcial ou total. Além disso, com a elaboração de curvas de lactação, pode-se prever antecipadamente as cabras potencialmente mais produtivas de um rebanho (Gall, 1981), facilitando a tomada de decisões sobre o descarte dos animais.

Segundo Wood (1980), o conhecimento da curva de lactação é necessário para determinar o manejo nutricional e reprodutivo de animais em lactação, por estimação da produção total por lactação, do pico de produção e da persistência de lactação. Diante deste contexto, é importante estabelecer parâmetros das curvas de lactação que melhor se ajustem à produção de leite em caprinos leiteiros, observando as diferenças

\footnotetext{
${ }^{1}$ Parte da dissertação de Mestrado do segundo autor.

${ }^{2}$ Professora Adjunto da Faculdade de Agronomia e Veterinária - FAV - UnB, Brasília - DF. E.mail: concepta@unb.br

3 Professor da Faculdade da Terra de Brasília (FTB) e Doutorando da Faculdade de Saúde - UnB.

${ }^{4}$ Pesquisador da Embrapa - Recursos Genéticos e Biotecnologia - Brasília - DF.
} 
entre raças e rebanhos, bem como os efeitos do ambiente nesses parâmetros.

A variação dos parâmetros que determinam a forma da curva de lactação pode sofrer influência das condições ambientais e, conseqüentemente, afetar a produção de leite (Gonçalves et al., 1997). Entretanto, as curvas de lactação de um rebanho proporcionam melhor entendimento sobre o índice produtivo do rebanho leiteiro, nas condições ambientais proporcionadas. McManus et al. (1997) relataram que muitos fatores podem afetar a produção total de leite em uma única lactação.

Os principais fatores que podem influenciar os níveis de produção e as curvas de lactação de uma cabra são: raça, estação do parto, idade da cabra, número de crias por parto, ambiente e alimentação (Morand-Fehr \& Sauvant, 1980; Gall, 1981; Gipson \& Grossman, 1990; Wahome et al., 1994; Ruvuna et al., 1995).

O objetivo deste trabalho foi avaliar os fatores ambientais e genéticos que influenciam os parâmetros da curva de lactação em cabras no Distrito Federal.

\section{Material e Métodos}

Os dados analisados neste trabalho foram coletados em duas propriedades no Distrito Federal, no período de 1995 a 1997. Os animais utilizados foram 104 da raça Saanen, 77 da raça Parda Alpina, 15 da raça Toggenburg e 19 mestiços, distribuídos nos dois criatórios.

O sistema de exploração nos dois capris era o confinamento e as instalações eram constituídas de piso ripado suspenso do solo. A alimentação fornecida consistia de volumoso com capim-elefante ou silagem de milho, de acordo com a época do ano. Além disso, os animais receberam um concentrado com mínimo de $20 \%$ de proteína bruta, além de quantidades que variaram de 400 a $600 \mathrm{~g} / \mathrm{dia}$ e mais 100 a $200 \mathrm{~g} / \mathrm{dia}$, fornecidos para cada quilo de leite produzido. Mistura mineral e a água foram fornecidas à vontade.

Os dados foram constituídos de pesagem do leite $(\mathrm{kg})$ a cada trinta dias, realizadas em duas ordenhas diárias, com intervalos de 8 a 10 horas, por 36 meses (janeiro de 1995 a dezembro de 1997).

Foram usadas quatro funções algébricas com a finalidade de se determinar, a partir dos respectivos valores do coeficiente de determinação $\left(R^{2}\right)$, o modelo que melhor se ajustasse aos dados, visando ao estudo da forma da curva de lactação, pico, persistência e produção total de leite.

R. Bras. Zootec., v.32, n.6, p.1614-1623, 2003 (Supl. 1)
As funções utilizadas foram as seguintes: Quadrática (Q):

$$
Y_{n}=a+b_{n}+c^{2}
$$

em que: Gama $(G): Y_{n}=a \cdot n^{b} \cdot e^{-c n}$, utilizada por Wood (1967) (nessa equação não linear o tempo em que ocorre o pico é estimado, como $\mathrm{n}=\mathrm{b} / \mathrm{c}$ ); Linear Hiperbólica (LH):

$$
\mathrm{Y}_{\mathrm{n}}=\mathrm{a}+\mathrm{b}_{\mathrm{n}}+\mathrm{c} / \mathrm{n}
$$

utilizada por Bianchini Sobrinho (1988) e equação descrita por Jenkins \& Ferrel (1984) (JF):

$$
\mathrm{Y}_{\mathrm{n}}=\mathrm{a} / \mathrm{ne}^{\mathrm{bn}}
$$

em que: $\mathrm{Y}_{\mathrm{n}}$ é a produção de leite diária na semana $\mathrm{n}$; $a$, a produção média no início da lactação; $b$, taxa média de ascensão; e $c$, taxa média de declínio da produção após o pico.

Foram encontrados, por intermédio dos métodos não lineares do programa "Table Curve" da companhia Jandel Scientific, os coeficientes de determinação $\left(\mathrm{R}^{2}\right)$ e os parâmetros $a, b$ e $c$ das curvas de lactação. Foram usados registros de 299 lactações, obtendo-se 290 curvas pela função Quadrática, 291 curvas pela função linear hiperbólica, 63 curvas pela função Jenkins e Ferrel. Os parâmetros a, b e c, foram estimados pelo método dos quadrados mínimos, através de um modelo estatístico linear de classificação múltipla, com auxílio computacional do programa de análise estatísticas Statistical Analysis System (SAS, 1985) usando os procedimentos GLM (General Linear Model) e CORR (Correlação), envolvendo os efeitos fixos de capril, raça, mês e ano do parto, tipo de parto, sexo do cabrito. O peso de cobertura da cabra foi utilizado como covariável. Os parâmetros das curvas foram correlacionados com intervalo de parto (IP), idade ao primeiro parto (IPP), persistência da lactação (PL) e produção de leite total (PLT).

Como os controles de leite foram realizados mensalmente, o cálculo da PLT foi feito através de uma equação, como segue:

PLT $(\mathrm{kg})=$ Cont. $1 \mathrm{x}$ dias $0-1+\frac{\text { Cont. } 1+\text { Cont. } 2}{2} \mathrm{x}$ dias $1-2+\ldots .+$
$\frac{\text { Cont.n-1+Cont.n }}{2} \mathrm{x}$ dias $(\mathrm{n}-1)-\mathrm{n}+$ Cont. $\mathrm{n} \mathrm{x}$ dias $(\mathrm{n}+1) \ldots \ldots \ldots . .(1)$ em que: $\mathrm{kg}$ de leite $=$ total de leite produzido na lactação; Cont. 1...n = número de controle; Dias 1$2 \ldots(\mathrm{n}-1)-\mathrm{n}=$ número de dias entre o controle 1 e 2 até o período entre os controles $n-1$ até n; Dias 0 1 = número de dias entre o início da lactação e o primeiro controle; e Dias $n+1=$ número de dias entre o último controle e o encerramento da lactação.

Em adição, ao cálculo da PLT (equação 1), foi calculada também a área das diferentes funções, pela 
integral de cada curva (ACQ, ACLH e ACJF para as curvas Quadrática, Linear Hiperbólica e Jenkins e Ferrel respectivamente). O cálculo da duração da lactação consistiu no número de dias transcorrido entre o início e o término da lactação.

O modelo estatístico geral utilizado no procedimento GLM foi o seguinte:

$$
\begin{gathered}
Y_{i j k l m n}=m+C_{i}+R_{j}+M_{k}+A_{1}+T_{m}+S_{n}+b_{1} \\
\left(P_{i j k l m n}-P\right)+b_{2}\left(P_{i j k l m n}-P\right)^{2}+e_{i j k l m n}
\end{gathered}
$$

em que: $\mathrm{Y}_{\mathrm{ijklmn}}=$ característica; $\mathrm{m}=$ constante inerente aos dados; $\mathrm{C}_{\mathrm{i}}=$ efeito fixo do iésima capril, $\mathrm{i}=1,2$; $\mathrm{R}_{\mathrm{j}}=$ efeito fixo da $\mathrm{j}$ ésima raça, $\mathrm{j}=$, Saanen, Parda Alpina e Toggenburg; $M_{k}=$ efeito fixo do k ésimo mês do parto, $\mathrm{k}=$ janeiro a dezembro; $\mathrm{A}_{1}=$ efeito fixo do lésimo ano do parto, 1 = 1995, 1996, 1997; $\mathrm{T}_{\mathrm{m}}=$ efeito fixo do $\mathrm{m}$ ésimo tipo de parto, $\mathrm{m}=$ simples e múltiplo; $\mathrm{S}_{\mathrm{n}}=$ efeito fixo do $\mathrm{n}$ ésimo sexo da cria, $\mathrm{n}=$ macho e fêmea; $b_{1}=$ coeficiente linear de regressão de peso da cabra à cobertura; $b_{2}=$ coeficiente quadrática de regressão de peso da cabra à cobertura; $\mathrm{P}_{\mathrm{ijk} k \mathrm{mn}}=$ peso da cabra à cobertura; $\mathrm{e}_{\mathrm{ijklmn}}=$ erro aleatório, normal, independentemente distribuído com médio zero e variância $\sigma^{2}$, associado a cada observação.

\section{Resultados e Discussão}

A análise de variância para função $\mathrm{Q}\left(\mathrm{R}^{2}, a, b\right.$ e $\left.c\right)$ é apresentada na Tabela 1 e revela diferenças significativas $(\mathrm{P}<0,05)$ para o sexo do cabrito sobre o coeficiente de determinação. Houve influência significativa $(\mathrm{P}<0,05)$ da raça e do tipo de parto sobre o parâmetro $a$ (produção média no início da lactação) nas curvas de lactação. Assim como, o tipo de parto influenciou o parâmetro $b$ (taxa média de ascensão de produção). Já o parâmetro $c$ (taxa média de declínio da produção após o pico) foi influenciado significativamente pelo mês do parto $(\mathrm{P}<0,05)$. As outras características estudadas não apresentaram influência sobre os parâmetros da curva.

Consta na Tabela 2 a análise de variância para os parâmetros da função $\mathrm{LH}\left(\mathrm{R}^{2}, a, b\right.$ e $\left.c\right)$, revelando que a raça influenciou os parâmetros $a$ e $c(\mathrm{P}<0,001$ e $\mathrm{P}<0,05$, respectivamente). Entretanto, não foram observados os mesmos efeitos para os parâmetros $b$ e $\mathrm{R}^{2}$. O mês do parto apresentou influência significativa nos parâmetros $a$ e $c(\mathrm{P}<0,01$ e $\mathrm{P}<0,001)$ e o tipo de parto influenciou os parâmetros $b$ e $c(\mathrm{P}<0,05$ e $\mathrm{P}<0,001)$ respectivamente.

$\mathrm{Na}$ função JF, os efeitos considerados na análise de variância (Tabela 3) não apresentaram influência nos parâmetros da curva de lactação, indicando que estes não interferiram na forma da curva. Provavelmente, isto ocorreu devido aos coeficientes de variação serem nulos (zero).

$\mathrm{Na}$ análise estatística observou-se que a função Gama não convergiu. Também não foi apresentado o número máximo de curvas (299 lactações) para as funções estudadas, uma vez que nem todas as funções

Tabela 1 - Resumo da análise de variância para função Quadrática $\left(R^{2}, a, b\right.$ e $\left.c\right)$

\begin{tabular}{|c|c|c|c|c|}
\hline \multirow[b]{2}{*}{$\begin{array}{l}\text { Fonte de variação } \\
\text { Source of variation }\end{array}$} & \multicolumn{4}{|c|}{$\begin{array}{l}\text { Quadrado médio } \\
\text { Mean square }\end{array}$} \\
\hline & $\mathrm{R}^{2}$ & $a$ & $b$ & $c$ \\
\hline $\begin{array}{l}\text { Capril } \\
\text { Farm }\end{array}$ & 0,000 & 0,061 & 0,000 & 4,040 \\
\hline $\begin{array}{l}\text { Raça } \\
\text { Breed }\end{array}$ & 0,094 & $2,182 *$ & 0,0001 & 16,851 \\
\hline $\begin{array}{l}\text { Mês do parto } \\
\text { Month of kidding }\end{array}$ & 0,033 & 0,761 & 0,0002 & $22,247^{*}$ \\
\hline $\begin{array}{l}\text { Ano do parto } \\
\text { Year of kidding }\end{array}$ & 0,122 & 2,181 & 0,000 & 12,244 \\
\hline $\begin{array}{l}\text { Tipo de parto } \\
\text { Type of kidding }\end{array}$ & 0,026 & $3,229 *$ & $0,0007^{*}$ & 12,537 \\
\hline $\begin{array}{l}\text { Sexo do cabrito } \\
\text { Sex of kid }\end{array}$ & $0,378^{*}$ & 0,452 & 0,000 & 2,687 \\
\hline $\begin{array}{l}\text { Peso de cobertura } \\
\text { Weight at insemination }\end{array}$ & 0,010 & 0,880 & 0,000 & 5,724 \\
\hline $\begin{array}{l}\mathrm{R}^{2} \\
\mathrm{CV}(\%)\end{array}$ & $\begin{array}{r}0,41 \\
33,42\end{array}$ & $\begin{array}{r}0,45 \\
40,23\end{array}$ & $\begin{array}{r}0,53 \\
188,44\end{array}$ & $\begin{array}{r}0,49 \\
201,07\end{array}$ \\
\hline
\end{tabular}

Table 1 - Summary of the analysis of variance for the Quadratic function $\left(R^{2}, a, b\right.$ and $\left.c\right)$

* $(\mathrm{P}<0,05)$ e CV $(\%)=$ coeficiente de variação (coefficient of variation). 
Tabela 2 - Resumo da análise de variância para função Linear Hiperbólica $\left(\mathrm{R}^{2}, a, b\right.$ e $\left.c\right)$

Table 2 - Summary of the analysis of variance for the Linear Hiperbolic function $\left(R^{2}, a, b\right.$ and $\left.c\right)$

\begin{tabular}{|c|c|c|c|c|}
\hline \multirow[b]{2}{*}{$\begin{array}{l}\text { Fonte de variação } \\
\text { Source of variation }\end{array}$} & \multicolumn{4}{|c|}{$\begin{array}{l}\text { Quadrado médio } \\
\text { Mean square }\end{array}$} \\
\hline & $\mathrm{R}^{2}$ & $a$ & $b$ & $c$ \\
\hline $\begin{array}{l}\text { Capril } \\
\text { Farm }\end{array}$ & 0,000 & 0,875 & 0,000 & 546,64 \\
\hline $\begin{array}{l}\text { Raça } \\
\text { Breed }\end{array}$ & 0,063 & $7,029 * * *$ & 0,000 & $2168,53^{*}$ \\
\hline $\begin{array}{l}\text { Mês do parto } \\
\text { Month of kidding }\end{array}$ & 0,038 & $3,196^{* *}$ & 0,000 & $2248,50 * * *$ \\
\hline $\begin{array}{l}\text { Ano do parto } \\
\text { Year of kidding }\end{array}$ & 0,113 & 0,661 & 0,000 & 481,63 \\
\hline $\begin{array}{l}\text { Tipo de parto } \\
\text { Type of kidding }\end{array}$ & 0,010 & 2,498 & $0,00008^{*}$ & $5521,52 * * *$ \\
\hline $\begin{array}{l}\text { Sexo do cabrito } \\
\text { Sex of kid }\end{array}$ & 0,043 & 0,048 & 0,000 & 38,60 \\
\hline $\begin{array}{l}\text { Peso de cobertura } \\
\text { Weight at insemination }\end{array}$ & 0,023 & 0,396 & 0,000 & 452,36 \\
\hline $\begin{array}{l}\mathrm{R}^{2} \\
\mathrm{CV}(\%)\end{array}$ & $\begin{array}{r}0,31 \\
38,28\end{array}$ & $\begin{array}{r}0,63 \\
30,31\end{array}$ & $\begin{array}{r}0,46 \\
73,19\end{array}$ & $\begin{array}{r}0,66 \\
126,59\end{array}$ \\
\hline
\end{tabular}

* $(\mathrm{P}<0,05)$ e CV $(\%)=$ coeficiente de variação (coefficient of variation).

utilizadas convergiram ou não foram possíveis encontrar resultados devidos aos diferentes parâmetros inicias.

Nas Tabelas 4 e 5, estão apresentadas as médias dos diferentes parâmetros para as variáveis estudadas nas funções Q e LH, respectivamente.

Foram observadas diferenças entre raças, mês do parto e tipo de parto em alguns parâmetros nas funções Q e LH (Tabelas 1, 2, 4 e 5, respectivamente). A raça Parda Alpina apresentou maior produção de leite inicial, seguida pela raça Saanen, quando ajustado pela função $\mathrm{LH}$, porém, não houve diferença entre estas raças, quando ajustada pela curva Q. O declínio após do pico apresentou comportamento similar na curva Q, enquanto na curva LH o declínio foi maior na Alpina, seguida da Saanen e mestiços, sendo a Toggenburg a raça que apresentou o menor declínio. $\mathrm{Na}$ curva $\mathrm{Q}$, as lactações iniciadas nos meses de julho-setembro (Tabela 4) apresentaram maiores c, mostrando tendência de que estas lactações tiveram seus picos ( \pm 2 meses após parto) nos meses que coincidiram com o início da estação de chuva, em que a qualidade do volumoso é melhor, coincidindo com observações de outros autores (Mourad, 1992, Gonçalves et al., 2001). Neste caso, as maiores necessidades nutricionais, bem como a elevação da temperatura nesta época do ano, que pode comprometer a ingestão de alimentos, possivelmente foram superadas pela qualidade do alimento volumoso em oferta.

O comportamento do mês do parto não mostrou tendência para as duas curvas estudadas. A influência

Tabela 3 - Resumo da análise de variância para função Jenkins e Ferrel $\left(\mathrm{R}^{2}\right.$, $a$ e $\left.b\right)$

Table 3 - Summary of the analysis of variance for the Jenkins e Ferrel function $\left(R^{2}\right.$, $a$ and $\left.b\right)$

\begin{tabular}{|c|c|c|c|}
\hline \multirow[b]{2}{*}{$\begin{array}{l}\text { Fonte de variação } \\
\text { Source of variation }\end{array}$} & \multicolumn{3}{|c|}{$\begin{array}{l}\text { Quadrado médio } \\
\text { Mean square }\end{array}$} \\
\hline & $\mathrm{R}^{2}$ & $A$ & $b$ \\
\hline Capril & 0,057 & 1453,83 & 0,0000 \\
\hline Farm & & & \\
\hline $\begin{array}{l}\text { Raça } \\
\text { Breed }\end{array}$ & 0,134 & 3200,51 & 0,0000 \\
\hline $\begin{array}{l}\text { Mês do parto } \\
\text { Month of kidding }\end{array}$ & - & - & 0,0000 \\
\hline $\begin{array}{l}\text { Ano do parto } \\
\text { Year of kidding }\end{array}$ & 0,013 & 529,18 & 0,0000 \\
\hline $\begin{array}{l}\text { Tipo de parto } \\
\text { Type of kidding }\end{array}$ & - & 2336,73 & 0,0000 \\
\hline $\begin{array}{l}\text { Sexo do cabrito } \\
\text { Sex of kid }\end{array}$ & - & - & - \\
\hline $\begin{array}{l}\text { Peso de cobertura } \\
\text { Weight at insemination }\end{array}$ & - & - & - \\
\hline $\mathrm{R}^{2}$ & 1,000 & 1,000 & 1,000 \\
\hline CV $(\%)$ & 0 & 0 & 0 \\
\hline
\end{tabular}


Tabela 4 - Média de quadrados mínimos das estimativas dos parâmetros $\left(R^{2}, Q a, Q b\right.$ e $\left.Q c\right)$ da função quadrática, conforme as variáveis analisadas

Table 4 - Minimum squares means for estimates of the parameters of the quadratic function, $\left(R^{2}, Q a, Q b\right.$ and $\left.Q c\right)$, according to the analyzed variable effects

\begin{tabular}{|c|c|c|c|c|}
\hline \multirow[t]{2}{*}{$\begin{array}{l}\text { Efeito } \\
\text { Effect }\end{array}$} & $\mathrm{R}^{2}$ & $\mathrm{Q} a(\mathrm{~kg})$ & $\mathrm{Q} b(\mathrm{~kg})$ & $\mathrm{Q} c(\mathrm{~kg})$ \\
\hline & \multicolumn{4}{|c|}{$\begin{array}{l}\text { Média } \\
\text { Average }\end{array}$} \\
\hline \multicolumn{5}{|l|}{ Capril } \\
\hline \multicolumn{5}{|l|}{ Farm } \\
\hline 1 & 0,73 & 2,36 & 0,006 & $-1,51$ \\
\hline 2 & 0,79 & 2,46 & 0,006 & $-0,63$ \\
\hline \multicolumn{5}{|l|}{ Raça } \\
\hline \multicolumn{5}{|l|}{ Breed } \\
\hline $\begin{array}{l}\text { Saanen } \\
\text { Parda Alpina }\end{array}$ & $\begin{array}{l}0,64 \\
0,80\end{array}$ & $\begin{array}{l}2,51^{\mathrm{a}} \\
2,56^{\mathrm{a}}\end{array}$ & $\begin{array}{l}0,006 \\
0,006\end{array}$ & $\begin{array}{l}-1,73 \\
-1,51\end{array}$ \\
\hline Toggenburg & 0,77 & $2,23^{b}$ & $-0,001$ & $-0,44$ \\
\hline Mestiços & 0,86 & $1,17^{\mathrm{c}}$ & 0,018 & $-3,63$ \\
\hline \multicolumn{5}{|l|}{ Mês do parto } \\
\hline \multicolumn{5}{|l|}{ Month of kidding } \\
\hline $\begin{array}{l}\text { Janeiro } \\
\text { January }\end{array}$ & 0,73 & 2,17 & 0,003 & $0,11^{\mathrm{a}}$ \\
\hline Fevereiro & 0,53 & 1,82 & 0,002 & $-5,74^{b}$ \\
\hline \multicolumn{5}{|l|}{ February } \\
\hline Março & 0,78 & 2,16 & $-0,002$ & $-2,41^{\mathrm{c}}$ \\
\hline \multicolumn{5}{|l|}{ March } \\
\hline Abril & 0,63 & 2,07 & 0,014 & $-2,44^{\mathrm{c}}$ \\
\hline \multicolumn{5}{|l|}{ April } \\
\hline Maio & 0,71 & 2,72 & 0,011 & $-6,95^{\mathrm{b}}$ \\
\hline \multicolumn{5}{|l|}{ May } \\
\hline Junho & 0,83 & 2,58 & 0,007 & $-0,67^{d}$ \\
\hline \multicolumn{5}{|l|}{ June } \\
\hline Julho & 0,84 & 3,32 & $-0,004$ & $0,98^{\mathrm{a}}$ \\
\hline \multicolumn{5}{|l|}{ July } \\
\hline Agosto & 0,84 & 1,56 & 0,023 & $0,51^{\mathrm{a}}$ \\
\hline \multicolumn{5}{|l|}{ August } \\
\hline Setembro & 0,65 & 2,74 & $-0,007$ & $0,81^{\mathrm{a}}$ \\
\hline \multicolumn{5}{|l|}{ September } \\
\hline Outubro & 0,92 & 2,75 & 0,008 & $-4,55^{b}$ \\
\hline \multicolumn{5}{|l|}{ October } \\
\hline Novembro & 0,73 & 2,48 & 0,007 & $-2,27^{\mathrm{c}}$ \\
\hline \multicolumn{5}{|l|}{ November } \\
\hline Dezembro & - & - & - & - \\
\hline \multicolumn{5}{|l|}{ December } \\
\hline \multicolumn{5}{|l|}{$\begin{array}{l}\text { Ano do parto } \\
\text { Year of kidding }\end{array}$} \\
\hline \multicolumn{5}{|l|}{ Year of kidding } \\
\hline 1995 & 0,67 & 2,02 & 0,002 & $-1,19$ \\
\hline 1996 & 0,76 & 2,38 & 0,006 & $-1,67$ \\
\hline 1997 & 0,69 & 2,43 & 0,008 & $-1,82$ \\
\hline \multicolumn{5}{|l|}{ Tipo de parto } \\
\hline Type of parturition & & & & \\
\hline Simples & 0,75 & $2,06^{\mathrm{a}}$ & $0,001^{\mathrm{a}}$ & $-1,89$ \\
\hline Single & & & & \\
\hline Duplo & 0,71 & $2,60^{\mathrm{b}}$ & $0,013^{b}$ & $-1,87$ \\
\hline Twin & & & & \\
\hline Triplo & 0,80 & $2,97^{\mathrm{c}}$ & $-0,007^{\mathrm{c}}$ & 3,36 \\
\hline Triple & & & & \\
\hline
\end{tabular}

Médias, nas colunas, seguidas de letras diferentes são diferentes $(P<0,05)$ pelo teste de Tukey.

Means, in the columns, followed by different letters are different $(P<.05)$ by Tukey test. 
Tabela 5 - Média de quadrados mínimos das estimativas dos parâmetros ( $R^{2}$, LHa, LHb e LHc) da função linear hiperbólica, conforme as variáveis analisadas

Table 5 - Minimum squares means for estimates of the parameters of the linear hyperbolic function $\left(R^{2}, L H a, L H b\right.$ and $\left.L H c\right)$, according to the analyzed variable effects

\begin{tabular}{|c|c|c|c|c|}
\hline Efeito & $\mathrm{R}^{2}$ & $\mathrm{LH} a(\mathrm{~kg})$ & $\mathrm{LH} b(\mathrm{~kg})$ & $\mathrm{LH} c(\mathrm{~kg})$ \\
\hline & & & & \\
\hline Capril & & & & \\
\hline Farm & & & & \\
\hline 1 & 0,70 & 3,45 & $-0,007$ & $-18,64$ \\
\hline 2 & 0,76 & 3,74 & $-0,009$ & $-32,68$ \\
\hline Raça & & & & \\
\hline Breed & & & & \\
\hline Saanen & 0,62 & $3,47^{\mathrm{a}}$ & $-0,007$ & $-14,74^{\mathrm{a}}$ \\
\hline Parda Alpina & 0,79 & $4,03^{b}$ & $-0,009$ & $-31,86^{b}$ \\
\hline Toggenburg & 0,72 & $2,62^{\mathrm{c}}$ & $-0,004$ & $-7,98^{\mathrm{c}}$ \\
\hline Mestiços & 0,78 & $2,69^{c}$ & $-0,006$ & $-18,79^{\mathrm{a}}$ \\
\hline Mês do parto & & & & \\
\hline Month of kidding & & & & \\
\hline Janeiro & 0,68 & $2,73^{\mathrm{a}}$ & $-0,005$ & $-5,259^{a}$ \\
\hline January & & & & \\
\hline Fevereiro & 0,57 & $2,36^{\mathrm{a}}$ & $-0,004$ & $-5,310^{a}$ \\
\hline February & & & & \\
\hline Março & 0,75 & $2,69^{\mathrm{a}}$ & $-0,007$ & $-14,73^{b}$ \\
\hline March & & & & \\
\hline Abril & 0,65 & $4,41^{b}$ & $-0,011$ & $-53,72^{\mathrm{c}}$ \\
\hline April & & & & \\
\hline Maio & 0,75 & $4,90^{\mathrm{b}}$ & $-0,012$ & $-41,92^{\mathrm{d}}$ \\
\hline May & & & & \\
\hline Junho & 0,78 & $3,76^{\mathrm{ab}}$ & $-0,010$ & $-19,65^{b}$ \\
\hline June & & & & \\
\hline Julho & 0,83 & $3,15^{\mathrm{a}}$ & $-0,006$ & $-3,88^{\mathrm{a}}$ \\
\hline July & & & & \\
\hline Agosto & 0,84 & $4,24^{\mathrm{b}}$ & $-0,012$ & $-4,86^{\mathrm{a}}$ \\
\hline August & & & & \\
\hline Setembro & 0,65 & $2,00^{\mathrm{a}}$ & $-0,002$ & $21,45^{\mathrm{e}}$ \\
\hline September & & & & \\
\hline Outubro & 0,81 & $3,97^{\mathrm{ab}}$ & $-0,008$ & $-19,06^{\mathrm{b}}$ \\
\hline October & & & & \\
\hline Novembro & 0,67 & $3,78^{a b}$ & $-0,007$ & $-24,04^{b}$ \\
\hline November & & & & \\
\hline Dezembro & - & - & - & - \\
\hline December & & & & \\
\hline Ano do parto & & & & \\
\hline Year of kidding & & & & \\
\hline 1995 & 0,63 & 2,59 & $-0,003$ & $-5,77$ \\
\hline 1996 & 0,72 & 3,35 & $-0,007$ & $-15,61$ \\
\hline 1997 & 0,69 & 3,92 & $-0,009$ & $-32,35$ \\
\hline Tipo de parto & & & & \\
\hline Type of parturition & & & & \\
\hline Simples & 0,70 & 3,15 & $-0,010^{\mathrm{a}}$ & $-34,02^{\mathrm{a}}$ \\
\hline Single & & & & \\
\hline Duplo & 0,70 & 3,24 & $-0,005^{b}$ & $-9,27^{b}$ \\
\hline Twin & & & & \\
\hline Triplo & 0,81 & 3,90 & $-0,007^{\mathrm{c}}$ & $-14,45^{\mathrm{c}}$ \\
\hline Triple & & & & \\
\hline
\end{tabular}

Médias, nas colunas, seguidas de letras diferentes são diferentes $(P<0,05)$ pelo teste de Tukey. Means, in the columns, followed by different letters are different $(P<.05)$ by Tukey test.

R. Bras. Zootec., v.32, n.6, p.1614-1623, 2003 (Supl. 1) 
da raça sobre os parâmetros da curva de lactação também foi observada por Gipson \& Grossman (1987), Ruvuna et al. (1995) e Lopes et al. (1995). Estes resultados foram contrários aos relatados por Câncio et al. (1992), em que as raças estudadas tiveram o mesmo comportamento, não influenciando os parâmetros da curva.

Em relação ao tipo de parto para a curva $\mathrm{LH}$, cabras que tiveram parto simples aumentaram a sua produção mais devagar até o pico, mas em compensação a sua produção teve declínio mais devagar. Com a curva Q, a produção inicial foi menor para as cabras de parto simples. Resultados próximos aos relatados neste estudo foram observados por Portolano et al. (1996). Resultados contrários foram obtidos por Macedo et al. (1990) e Wahome et al. (1994), em que este fator teve influência na forma da curva de lactação.

Os meses de maior produção inicial de leite foram distribuídos ao longo do ano, mas principalmente no fim da época seca quando ajustada pela curva LH. Resultados semelhantes foram relatados por inúmeros autores: Wood (1970, 1972 e 1980), Carles et al. (1987), Gipson \& Grossman (1989), Wahome et al. (1994), Lopes et al. (1995), Portolano et al. (1996) e Gonçalves et al. (1997). Gipson \& Grossman (1987), utilizando a função Gama incompleta, não verificaram influência da estação do parto sobre os parâmetros da curva de lactação.

Observa-se que o fato de a raça ter influenciado os parâmetros da curva pode ser atribuído à constituição genética dos rebanhos estudados. Quanto à influência do mês do parto, possivelmente essas variações foram decorrentes das diferenças ambientais ou mudanças de manejo, principalmente alimentar. Já o tipo de parto, as diferenças podem ser causadas por fatores fisiológicos, onde geralmente animais que têm partos múltiplos produzem mais leite do que aqueles com partos simples, influenciando alguns parâmetros das curvas.

Os resultados obtidos neste estudo mostram que o mês do parto influenciou alguns dos parâmetros. Tais resultados podem ser atribuídos às diferenças na alimentação, como, por exemplo, mudanças no manejo, na composição do concentrado ou na qualidade das forrageiras, bem como nas condições climáticas (precipitação pluviométrica, temperatura e umidade do ar) que exercem influência sobre o conforto do animal em confinamento.
Os valores estimados dos parâmetros das curvas de lactação são apresentados no Tabela 6. Em geral, os coeficientes de determinações das curvas $\left(\mathrm{R}^{2}\right)$ médios variam entre 46 e $79 \%$, com desvio-padrão alto, indicando a dificuldade em se encontrar uma curva para explicar todas as lactações. Observou-se que as funções Q e LH apresentaram $\mathrm{R}^{2}$ alto, variando entre 73 e $79 \%$, sendo, portanto, as equações que melhor se ajustaram aos dados.

Os gráficos das curvas estão nas Figuras 1 e 2. A função JF superestimou o pico da lactação, particularmente no criatório 1.
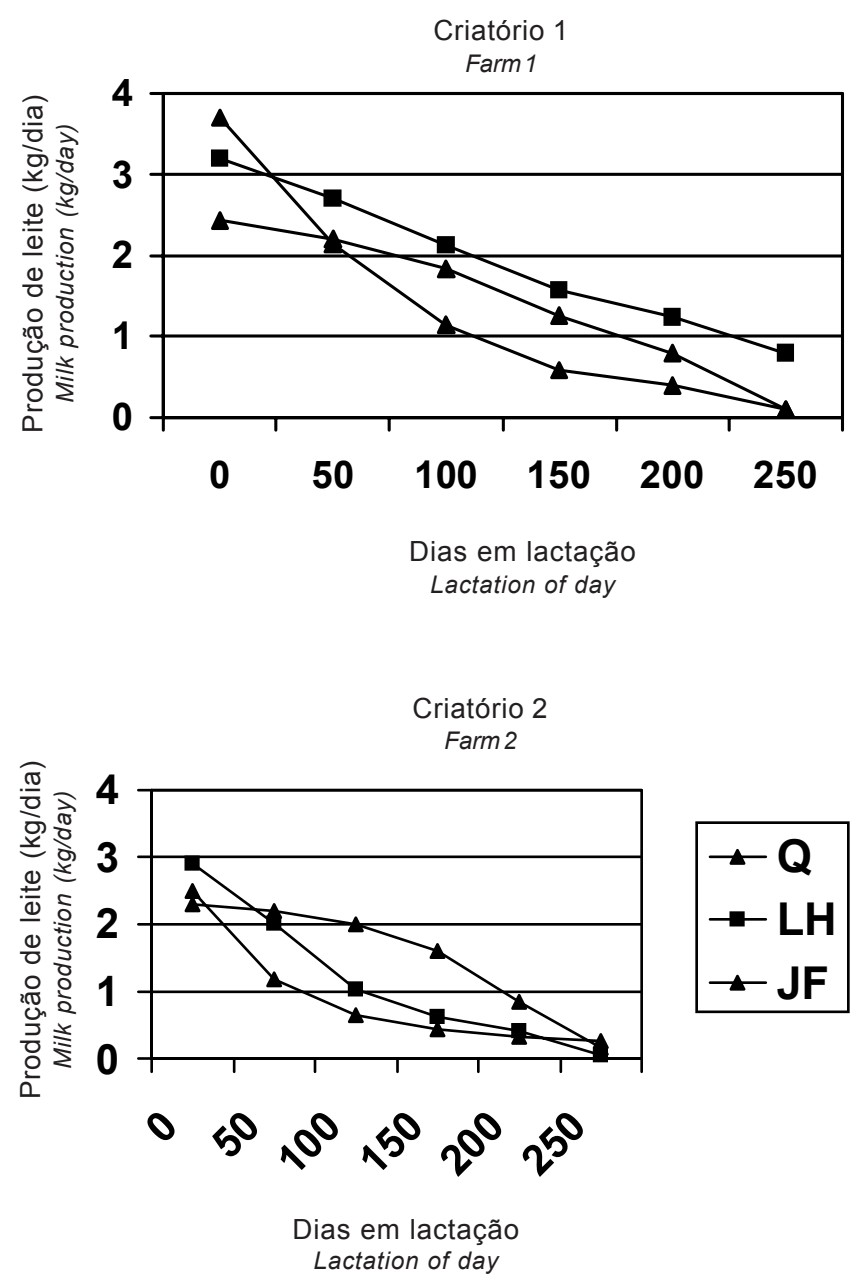

Figura 1 - Curvas de lactação ajustadas (Q-Quadrática; LH - Linear Hiperbólica; JF - Jenkins e Ferrel) para o criatórios 1 e 2 no Distrito Federal.

Figure 1 - Adjusted lactation curves (Q - Quadratic; LH Linear Hyperbolic; JF - Jenkins and Ferrel) for farm 1 and 2 in the Federal District, Brazil. 
Constatou-se que as interpretações biológicas dessas estimativas foram prejudicadas, pois não estão de acordo com a lactação normal, o que pode ter ocorrido em virtude das oscilações observadas. Estas oscilações podem ter influência das variações climáticas, de manejo e da alimentação, no decorrer dos anos estudados.

As funções Q e LH descrevem a taxa média de declínio da produção após o pico como a persistência da lactação (PL), que é o período da lactação que se mantém com altos níveis de produção de leite.

Observou-se, nas Figuras 1 e 2, que, em todas as curvas obtidas, as médias de produção de leite no início da lactação tiveram a mesma tendência apresentando os maiores níveis de produção. No entanto,

Tabela 6 - Médias das funções dos parâmetros e área (AC) das curvas quadrática, linear hiperbólica e Jenkins e Ferrel

Table 6 - Means of the paramaters and areas (AC) of quadratic, linear hiperbolic and Jenkins and Ferrel function

\begin{tabular}{lcc}
\hline & Capril 1 & Capril 2 \\
& Farm 1 & Farm 2 \\
\hline Variável & Média & Média \\
Average & Average \\
\hline Variable & 0,75 & 0,79 \\
$\mathrm{QR}^{2}$ & 2,436 & 2,612 \\
$\mathrm{Q} a(\mathrm{~kg})$ & $-0,007$ & $-0,011$ \\
$\mathrm{Q} b$ & $-1,52 \times 10^{-5}$ & $-1,9 \times 10^{-5}$ \\
$\mathrm{Q} c$ & 674,05 & 630,14 \\
$\mathrm{ACQ}(\mathrm{kg})$ & 0,73 & 0,78 \\
$\mathrm{LHR}$ & 3,29 & 2,99 \\
$\mathrm{~L} h a(\mathrm{~kg})$ & $-0,0076$ & $-0,019$ \\
$\mathrm{LH} b$ & $-1,69$ & $-0,72$ \\
$\mathrm{LH} c$ & 495,00 & 489,65 \\
$\mathrm{ACLH}(\mathrm{kg})$ & 0,46 & 0,52 \\
$\mathrm{JFR}$ & 93,42 & 70,60 \\
$\mathrm{~J} f a(\mathrm{~kg})$ & 5,80 & 9,00 \\
$\mathrm{JF} b$ & 681,40 & 571,16 \\
$\mathrm{ACJF}(\mathrm{kg})$ & &
\end{tabular}

Função Quadrática (Quadratic function) $=\left(R^{2}, Q a, Q b, Q c\right.$ e $\left.A C Q\right)$; Função linear hiperbólica (Linear hiperbolic function) $=\left(R^{2}\right.$, LHa, $\mathrm{LH} b, \mathrm{LH} c, \mathrm{ACLH}$ ); Função Jenkins e Ferrel (Jenkins and Ferrel function $)=\left(\mathrm{R}^{2}, \mathrm{JF} a, \mathrm{JF} b\right.$ e ACJF $)$. a taxa média de ascensão não representou bem o pico de produção de leite nas funções estudadas. A taxa média de declínio da produção ficou bem descrita em todas as funções.

Com base nos resultados deste estudo, apresentados na Tabela 6 e nas Figuras 1 e 2, concluiu-se que a função Q descreve melhor a curva de lactação do que as demais em todos os estádios. Na literatura revisada, não foram encontrados estudos semelhantes para caprinos, no entanto, em bovinos, McManus et al. (1997) relataram que a função Q também foi a que melhor se ajustou aos dados estudados.

As correlações entre os parâmetros a para as curvas Q e JF (Tabela 7), demonstraram a similaridade entre as estimativas deste parâmetro pelas duas curvas. Em termos de correlações entre os parâmetros das mesmas curvas, a correlação de a com b foi significativa, indicando que a menor produção inicial teve o menor pico de lactação, para todas as curvas. Os outros parâmetros não mostraram nenhuma tendência.

As correlações entre as características de produção e reprodução são em geral baixas, exceto para intervalo de partos com período de lactação, que é alta e negativa, indicando que as melhores produções de leite tiveram os piores intervalos de partos. As correlações entre os parâmetros das curvas e características de reprodução são em geral baixas. $\mathrm{O}$ parâmetro a para as funções JF e Q e b para a função JF com período de lactação foram altos e positivos, indicando que são bons preditores de produção de leite.

As correlações das áreas abaixo das curvas com a estimativa total de produção e leite foram mais altas para as curvas JF $(0,78)$ e LH $(0,65)$. A correlação entre estas duas áreas foi 0,83 . Estas mesmas áreas também tiveram correlações altas com os parâmetros a das curvas Q e JF. A área embaixo da curva $Q$ teve correlação alta com os parâmetros $b$ da curva Q, enquanto a da curva LH, alta e negativa com o parâmetro $c$ da curva LH. 


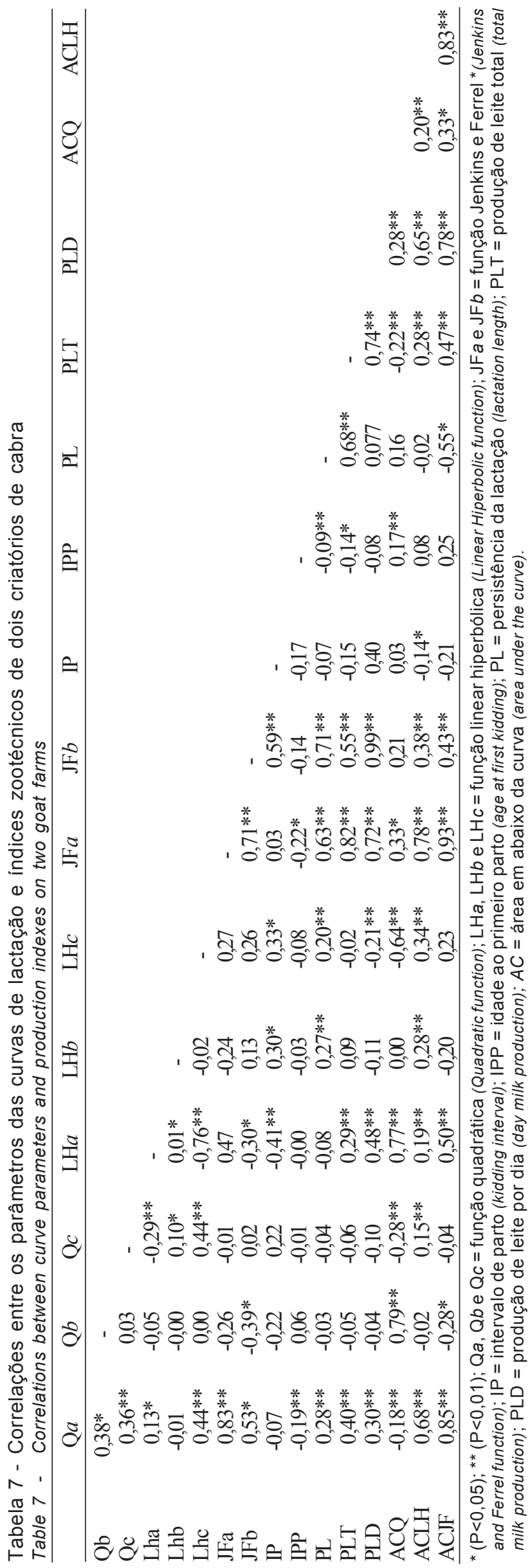

\section{Conclusões}

Os resultados mostraram coerência entre os dois criatórios. As correlações entre os parâmetros das curvas e índices zootécnicos variaram, porém, em geral, no parâmetro a (produção no início da lactação e produção de leite) foram médias e positivas. Qualquer uma das funções estudadas poderia representar a curva de lactação individual do rebanho, no entanto, neste estudo, a função que melhor expressou os parâmetros e o maior coeficiente de determinação na formação da curva foi a Quadrática. Os parâmetros das curvas Jenkins e Ferrel tiveram maior correlação com os níveis de produção leiteira calculada em várias maneiras.

\section{Agradecimento}

Ao Prof. João Batista Soares, pela ajuda com as equações matemáticas.

\section{Literatura Citada}

BIANCHINI-SOBRINHO, E.; DUARTE, F.A.M. Genetic and Environmental aspects of the linear hyperbolic lactation curve. Revista Brasileira de Genética, v.11, n.3, p.671678, 1988.

CÂNCIO, C.R.B.; CASTRO, R.S. de; COELHO, L.A. et al. Idade ao primeiro parto, intervalo entre partos e produção leiteria de cabras Saanen, Marota e mestiças em Alagoas. Pesquisa Agropecuária Brasileira, v.27, n.1, p.53-59, 1992.

CARLES, A.B.; WAHOME, R.G.; SCHWARTZ, H.J. Milk yields and the lactation curve in a herd of small east african goats on a semi-arid thornbush savannah. In: INTERNATIONAL CONFERENCE ON GOATS, 4., 1987, Brasília. Proceedings... Brasília, 1987. v.2, p.1456-1457.

GALL, C. Milk production. In: Goat production. New York: Academic Press, 1981. p.309-344.

GIPSON, T.A.; GROSSMAN, M. Lactation curves in dairy goats. In: INTERNATIONAL CONFERENCE ON GOATS, 4., 1987, Brasília. Proceedings... Brasília: 1987. v.2, p.14481449.

GIPSON, T.A.; GROSSMAN, M. Diphasic analysis of lactation curves in dairy goats. Journal of Dairy Science, v.72, p.1035-1044, 1989.

GIPSON, T.A.; GROSSMAN, M. Lactation curves in dairy goats: a review. Small Ruminant Research, v.3, p.383396, 1990.

GONÇALVES, T.M.; MARTINEZ, M.L.; MILAGRES, J.C. Curva de lactação na raça Gir. 2. Influência dos fatores de meio ambiente, estimativa de repetibilidade e herdabilidade para parâmetros da curva de lactação quadrática logarítmica. Revista Brasileira de Zootecnia, v.26, p.88-97, 1997.

GONÇALVES, H.C.; SILVA, M.A., WECHSLER, F.S.; et al. Fatores genéticos e de meio na produção de leite de caprinos leiteiros. Revista Brasileira de Zootecnia, v.30, n.3, p.719-729, 2001. 
GRAMINHA, C.V.; RESENDE, K.T.; RIBEIRO, S.D.A. et al. Estudo comparativo entre as curvas de produção real e a curva de produção teórica em cabras leiteiras. In. REUNIÃO ANUAL DA SOCIEDADE BRASILEIRA DE ZOOTECNIA, 33., 1996, Fortaleza. Anais... Fortaleza: Sociedade Brasileira de Zootecnia, 1996. p.552-553.

JENKINS, T.G.; FERREL, C.L. A note on lactation curves of crossbred cows. Animal Production, v.39, p.479-482, 1984.

LOPES, M.A.; VEIGA, R.D.; NEIVA, R.S. et al. Aplicação da função tipo gama no estudo da curva de lactação de vacas da raça Holandesa e suas mestiças Holandês x Sindhi. In: REUNIÃO ANUAL DA SOCIEDADE BRASILEIRA DE ZOOTECNIA, 32., 1995, Brasília. Anais... Brasília: Sociedade Brasileira de Zootecnia, 1995. p.643-644.

MACEDO, F.A.F.; MARTINS, E.N.; PRADO, I.N. et al. Comportamento da curva de lactação de cabras Sem Raça Definida. In. REUNIÃO ANUAL DA SOCIEDADE BRASILEIRA DE ZOOTECNIA, 27., 1990, Campinas. Anais... Campinas: Sociedade Brasileira de Zootecnia, 1990. p.513.

McMANUS, C.; GUTH, T.L.F.; SAUERESSIG, M.G. Curvas de lactação em gado holandês em confinamento total no DF. In: REUNIÃO ANUAL DA SOCIEDADE BRASILEIRA DE ZOOTECNIA, 34., 1997, Juiz de Fora. Anais... Juiz de Fora, 1997. p.74-76.

MORAND-FEHR, P.; SAUVANT, D. Composition and yield of goat milk as affected by nutritional manipulation. Journal of Dairy Science, v.63, p.1671-1680, 1980.

MOURAD, M. Effects of month of kidding, parity and litter size on milk yield of Alpine goats in Egypt. Small Ruminant Research, v.8, p.41-46, 1992.
PORTOLANO, B.; SPATAFORA, F.; BONO, G. et al. Application of the Wood model to lactation curves of Comisana sheep. Small Ruminant Research, v.24, p.7-13, 1996.

RUVUNA, F.; KOGI, J.K.; TAYLOR, J.F. et al. Lactation curves among crosses of Galla and East African with Toggenburg and Anglo Nubian goats. Small Ruminant Research, v.16, p1-6, 1995.

STATISTICAL ANALYSIS SYSTEM - SAS. User's guide: Statistics. Cary: 1985.

WAHOME, R.G.; CARLES, A.B.; SCHWARTZ, H.J. An analysis of the variation of the lactation curve of small east African goats. Small Ruminant Research, v.15, p.1-7, 1994.

WOOD, P.D.P. Algebraic model of the lactation curve in cattle. Nature, v.216, p.164-165, 1967.

WOOD, P.D.P. The relationship between the month of calving and milk production. Journal of Animal Production, v.12, n.2,p.253-259, 1970.

WOOD, P.D.P. A note on seasonal fluctuations in milk production. Journal of Animal Production, v.15, n.1, p.89-92, 1972.

WOOD, P.D.P. Breed variation in the shape of the lactation curve of cattle and their implications for efficiency. Journal of Animal Production, v.34, p.133-141, 1980.

Recebido em: 23/11/99

Aceito em: 02/04/03 